\title{
Transient Approach to Radiative Heat Transfer Free Convection Flow with Ramped Wall Temperature
}

\author{
R.R. Patra ${ }^{1}$, S. Das ${ }^{1}$, R.N. Jana ${ }^{1}$ and S.K. Ghosh ${ }^{2 \dagger}$ \\ ${ }^{1}$ Department of Applied Mathematics, Vidyasagar University, Midnapore 721 102, West Bengal, India \\ ${ }^{2}$ Department of Mathematics, Narajole Raj College,Narajole, Midnapore 721 211, West Bengal, India \\ †Corresponding Author Email: g_swapan2002@yahoo.com
}

(Received February 9, 2010; accepted July 13, 2010)

\begin{abstract}
The effect of radiation on natural convection incompressible viscous fluid near a vertical flat plate with ramped wall temperature has been studied. An analytical solution of the governing equation has been obtained by employing Laplace transform technique. It is examined that two different solutions for the fluid velocities, one valid for fluids of Prandtl number $(P r)$ different from $1+R a, R a$ being the radiation parameter and the other for which the Prandtl number equal to $1+R a$. The variations of velocities and fluid temperature are presented graphically. Furthermore, the radiative heat transfer on natural convection flow near a ramped plate temperature has been compared with the flow near a plate with the constant wall temperature. It is found that an increase in radiation parameter leads to rise the fluid velocity as well as temperature.
\end{abstract}

Keywords: Natural convection, Radiation, Ramped temperature, Isothermal plate and Stefan-Boltzman constant.

\section{NOMENCLATURE}

$\begin{array}{ll}c_{p} & \text { Specific heat at constant pressure } \\ g & \text { Acceleration due to gravity } \\ H & \text { Unit step function } \\ k^{\prime} & \text { Thermal conductivity } \\ k^{*} & \text { Spectral mean absorption } \\ & \text { coefficient of the medium } \\ N u & \text { Nusselt number } \\ \operatorname{Pr} & \text { Prandtl number } \\ q & \text { Radiative heat flux } \\ R a & \text { Radiation parameter } \\ T^{\prime} & \text { Temperature of the fluid } \\ T_{\infty} & \text { Temperature of the fluid } \\ t^{\prime} & \text { Time } \\ t_{0} & \text { Characteristic time } \\ u^{\prime} & \text { Velocity component in } x \text {-direction }\end{array}$

\section{INTRODUCTION}

Over the recent years an analyzing mode of radiationconvection heat transfer takes place in many practical applications of glass manufacturing, furnace technology, high temperature aerodynamics, fire dynamics and space craft reentry (1967). England and Emery (1969) have studied the thermal radiation effects of an optically thin gray gas bounded by a stationary

\author{
$u \quad$ Dimensionless velocity \\ $\left(x^{\prime}, y^{\prime}\right)$ Cartesian co-ordinates \\ $\beta \quad$ Coefficient of thermal expansion \\ $\sigma \quad$ Stefan-Boltzman constant \\ $\eta \quad$ Non-dimensionalized width of the channel \\ $\theta$ Dimensionless temperature \\ $v \quad$ Kinematic coefficient of viscosity \\ $\rho \quad$ Fluid density \\ $\tau \quad$ Non-dimensional time \\ $\tau_{0} \quad$ Skin friction at the plate $\eta=0$ \\ Subscripts \\ $w \quad$ Condition at wall \\ $\infty$ Condition at infinity \\ Superscripts \\ Differentiation with respect to $\eta$
}

plate. The hydromagnetic free convection flow with radiation heat transfer in a rotating and optically thin fluid has been investigated by Bestman and Adiepong (1988) and Naroua et al. (1998). Raptis and Perdikis (2003) have studied thermal radiation of an optically thin gray gas. Hossain and Takhar (1996) have studied the mixed convection flat plate boundary layer problem using Rosseland (diffusion) flow model. Takhar et al. (1996) have studied the radiation effects on MED free 
convection flow in a radiating gas past a semi-infinit vertical plate. Radiation effect on the flow past impulsively started infinite vertical plate with variable temperature was studied by Muthucumaraswamy and Ganesan (2003). Cheng (1965) highlighted the radiation-convective gas dynamic flow using a differential approximation. Cess (1966) presented a seminal study of radiating convective boundary layers with buoyancy effects using Rosseland diffusion approximation. Ghosh and Pop (2007) have considered thermal radiation of an optically thick gray gas in the presence of indirect natural convection by employing Rosseland approximation for diffusion concept of radiation heat transfer. Chandran et al. (2005) have studied the unsteady natural convection near a vertical plate with ramped wall temperature. Concerning to all these studies the orientation of our present problem is to study of radiation-convection heat transfer flow of an optically dense medium to boost-up effective temperature of a different Prandtl number. The aim of our present investigation is to analyze the unsteady radiation-convection flow resulting from the ramped temperature profile of the bounding plate. In an optically dense medium there arise a thin radiation layer on the border of the thermal boundary and the Prandtl number may have a significant relation with radiation parameter. Thus the variation of temperature may come into true for the case of a different Prandtl number due to the change of a different characteristics of an atomic gas. Although nuclear reaction does not involved in all these studies it is to be mentioned that a homogeneous reaction takes place to equal concentration of ions and electrons and the chemical reaction becomes ignored. Therefore, in a non- catalytic system, radiative heat transfer natural convection flow of a ramped wall temperature communicates a physical background of aerospace science for its optical measurement.

The motivation of our present investigation is to study of a laminar viscous flow of different Prandtl number near an infinite vertical flat plate of a heat generating fluid with reference to a radiation-convection heat source. An attempt has been made to analyze the ramped temperature at the wall $\left(t^{\prime}>0\right)$ to exert its fluid behaviour within the radiation boundary layer. It is assumed that a homogeneous reaction takes place in the direction of a radiation boundary layer flow of an optically dense medium. To recast a qualitative analysis of this problem it is essential to study of a transient approach on radiation-convection flow near a vertical plate with ramped wall temperature. The radiation heat transfer on natural convection flow near a ramped wall temperature has also been compared with the flow near the plate with constant temperature.

\section{Formulation of The Problem AND ITS SOLUTION}

We consider the unsteady flow of a viscous incompressible fluid of arbitrary Prandtl number (Pr) near an infinite vertical flat plate. Choose the cartesian co-ordinate system in such a way that $x^{\prime}$-axis is taken along the wall in a vertically upward direction and $y^{\prime}$ axis is normal to it into the fluid. Our aim is to analyze the radiative heat transfer of an unsteady free convection flow resulting from the ramped temperature profile of the bounding plate. At time $t^{\prime}<0$, both the fluid and plate are at rest and constant temperature $T_{\infty}^{\prime}$.

At time $t^{\prime}>0$, the temperature of the plate is raised or lowered to $T_{\infty}^{\prime}+\left(T_{w}^{\prime}-T_{\infty}^{\prime}\right) \frac{t^{\prime}}{t_{0}} \quad$ when $t^{\prime} \leq t_{0}$ and the constant temperature $T_{w}^{\prime}$ is maintained at $t^{\prime}>t_{0}$. Since the plate is infinite along $x^{\prime}$ - direction, all the physical variables are the function of $y^{\prime}$ and $t^{\prime}$ only. The flow is considered optically thick gray gas with natural convection and radiation. The radiative heat flux in the $x^{\prime}$-direction is considered negligible in comparison to $y^{\prime}$-direction (See Fig. 1).

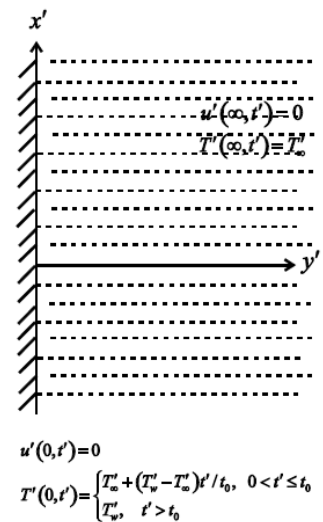

Fig. 1. Geometry of the problem

Under Boussinesq approximation, the momentum equation becomes

$\frac{\partial u^{\prime}}{\partial t^{\prime}}=v \frac{\partial^{2} u^{\prime}}{\partial y^{\prime 2}}+g \beta\left(T^{\prime}-T_{\infty}^{\prime}\right)$.

The energy equation becomes

$\frac{\partial T^{\prime}}{\partial t^{\prime}}=\frac{k}{\rho c_{p}} \frac{\partial^{2} T^{\prime}}{\partial y^{\prime 2}}-\frac{1}{\rho c_{p}} \frac{\partial q}{\partial y^{\prime}}$,

where $u^{\prime}$ is the velocity in the $x^{\prime}$-direction, $T^{\prime}$ - the temperature of the fluid, $g$-the acceleration due to gravity, $\beta$ - the coefficient of thermal expansion, $v$ the kinematic coefficient of viscosity, $\rho$ - the fluid density, $k$ - the thermal conductivity, $c_{p}$ - the specific heat at constant pressure and $q$ - the radiative heat flux.

The initial and boundary conditions are to be satisfied

$u^{\prime}=0, T^{\prime}=T_{\infty}^{\prime}$ for $y^{\prime} \geq 0$ and $t^{\prime} \leq 0$,

$u^{\prime}=0$ at $y^{\prime}=0$ at $t^{\prime}>0$,

$T^{\prime}=T_{\infty}^{\prime}+\left(T_{w}^{\prime}-T_{\infty}^{\prime}\right) \frac{t^{\prime}}{t_{0}}$ at $y^{\prime}=0$ for $t_{0} \geq t^{\prime}$,

$T^{\prime}=T_{w}^{\prime}$ at $y^{\prime}=0$ for $t^{\prime}>t_{0}$,

$u^{\prime} \rightarrow 0, T^{\prime} \rightarrow T_{\infty}^{\prime}$ as $y^{\prime} \rightarrow \infty$ for $t^{\prime}>0$.

We introduce dimensionless variables

$\eta=\frac{y^{\prime}}{\sqrt{v t_{0}}}, \tau=\frac{t^{\prime}}{t_{0}}, u=u^{\prime} \sqrt{\frac{t_{0}}{v}}, \theta=\frac{T^{\prime}-T_{\infty}^{\prime}}{T_{w}^{\prime}-T_{\infty}^{\prime}}$. 
The radiation heat flux can be found from Isachenko et al. (1969) and Brewster (1992) and its formula is derived from the diffusion concept of radiation heat transfer in the following way

$q=-\frac{4 \sigma}{3 k^{*}} \frac{\partial T^{\prime 4}}{\partial y^{\prime}}$,

where $\sigma$ is the Stefan-Boltzman constant and $k^{*}$ the spectral mean absorption coefficient of the medium.

Equations (1) and (2) together with Eq. (5) subject to Eq. (4) can be written in a dimensionless form

$\frac{\partial u}{\partial \tau}=\frac{\partial^{2} u}{\partial \eta^{2}}+\theta$

$\operatorname{Pr} \frac{\partial \theta}{\partial \tau}=(1+R a) \frac{\partial^{2} \theta}{\partial \eta^{2}}$

where $R a=\frac{16 \sigma T_{\infty}^{\prime 3}}{3 k^{*} k}$ is the radiation parameter and $\operatorname{Pr}=\frac{\rho v c_{p}}{k}$ is the Prandtl number.

The characteristic time $t_{0}$ can be express as

$t_{0}=\left[\frac{v}{g \beta\left(T_{w}^{\prime}-T_{\infty}^{\prime}\right)^{2}}\right]^{\frac{1}{3}}$.

The corresponding boundary conditions are

$u=0, \theta=0$ for $\eta \geq 0$ and $\tau \leq 0$,

$u=0$ at $\eta=0$ for $\tau>0$,

$\theta=\tau$ for $0<\tau \leq 1$,

$\theta=1$ for $\tau>1$,

$u \rightarrow 0, \theta \rightarrow 0$ as $\eta \rightarrow \infty$ for $\tau>0$.

By employing Laplace transform technique the Eqs. (6) and (7) can be solved by the defined $t_{0}$ in Eq. (8) subject to the boundary conditions (9) and the solutions for the resulting differential Eqs. (6) and (7) become

$$
\begin{aligned}
& \bar{u}(\eta, s)=\frac{(1+R a)\left(1-e^{-s}\right)}{(1+R a-P r) s^{3}}\left(e^{-\sqrt{\frac{P r s}{1+R a}} \eta}-e^{-\sqrt{s} \eta}\right), \\
& \bar{\theta}(\eta, s)=\left(\frac{1-e^{-s}}{s^{2}}\right) e^{-\sqrt{\frac{P r s}{1+R a}} \eta} .
\end{aligned}
$$

The exact solutions can be obtained by applying inverse Laplace transform of the Eqs. (10) and (11) for temperature and velocity distribution on shifting the $\tau$ axis and these turn into the following form

$$
\begin{aligned}
& \theta(\eta, \tau)=\theta_{1}(\eta, \tau)-\theta_{1}(\eta, \tau-1) H(\tau-1), \\
& u(\eta, \tau)=\frac{1+R a}{1+R a-\operatorname{Pr}}\left[u_{11}(\eta, \tau)-u_{12}(\eta, \tau)\right. \\
& \left.-\left\{u_{11}(\eta, \tau-1)-u_{12}(\eta, \tau-1)\right\} H(\tau-1)\right], \\
& \text { where } \\
& \theta(\eta, \tau)=\left(\tau+\frac{1}{2} \cdot \frac{P r \eta^{2}}{1+R a}\right) \operatorname{erfc}\left\{\frac{\eta \sqrt{P r}}{2 \sqrt{\tau(1+R a)}}\right\} \\
& -\sqrt{\frac{P r \tau}{\pi(1+R a)}} \eta e^{-\frac{P r}{4 \tau(1+R a)}},
\end{aligned}
$$

$$
\begin{aligned}
u_{11} & =\frac{1}{2}\left\{\frac{1}{12} \frac{\operatorname{Pr}^{2} \eta^{4}}{(1+R a)^{2}}+\frac{\operatorname{Pr} \tau \eta^{2}}{1+R a}+\tau^{2}\right\} \\
& \times \operatorname{erfc}\left\{\frac{\eta \sqrt{\operatorname{Pr}}}{2 \sqrt{\tau(1+R a)}}\right\} \\
& -\frac{1}{12} \eta \sqrt{\frac{\operatorname{Pr} \tau}{\pi(1+R a)}}\left(\frac{\operatorname{Pr} \eta^{2}}{1+R a}+10 \tau\right) e^{-\frac{P r}{4 \tau(1+R a)}}, \\
u_{12}= & \frac{1}{2}\left(\frac{1}{12} \eta^{4}+\tau \eta^{2}+\tau^{2}\right) \operatorname{erfc}\left(\frac{\eta}{2 \sqrt{\tau}}\right) \\
& -\frac{1}{12} \eta \sqrt{\frac{\tau}{\pi}}\left(\eta^{2}+10 \tau\right) e^{-\frac{\eta^{2}}{4 \tau}},
\end{aligned}
$$

where erfc $(x)$ being complementary error function and $H(\tau-1)$ is the unit step function. It is noticed that in the absence of radiation parameter $(R a=0)$, the solution (13) is identically satisfied with Chandran et al. (2005).

\subsection{Solution When Prandtl Number $P r=1$}

It has been observed from Eq. (13) that the solution for velocity was not valid for fluids of Prandtl number unity when $R a=0$. It is seen that the solution for temperature $\theta(\eta, \tau)$ is obtained from Eq. (12) by substituting $\operatorname{Pr}=1+R a$. However, in the case of $u(\eta, \tau)$, the solution has rederived starting from Eqs. (6) and (7). Following the same procedure as before, the solution for the velocity distribution turns into

$u(\eta, \tau)=u_{0}(\eta, \tau)-u_{0}(\eta, \tau-1) H(\tau-1)$,

where

$$
\begin{aligned}
u_{0}(\eta, \tau) & =\frac{\eta}{3} \sqrt{\frac{\tau}{\pi}}\left(\frac{\eta^{2}}{2}+2 \tau\right) e^{-\frac{\eta^{2}}{4 \tau}} \\
& -\frac{\eta^{2}}{2}\left(\frac{\eta^{2}}{6}+\tau\right) \operatorname{erfc}\left(\frac{\eta}{2 \sqrt{\tau}}\right)
\end{aligned}
$$

\subsection{Solution for Isothermal Plate}

To highlight the effects of ramped temperature distribution on the boundary of the flow we have solved the problem with constant wall temperature distribution. The temperature and the velocity for the flow near an isothermal, stationary plate can be expressed as

$$
\begin{aligned}
& \theta(\eta, \tau)=\operatorname{erfc}\left\{\frac{\eta \sqrt{P r}}{2 \sqrt{\tau(1+R a)}}\right\}, \\
& u(\eta, \tau)=\frac{1+R a}{1+\operatorname{Ra}-\operatorname{Pr}}\left[u_{1}(\eta, \tau)-u_{2}(\eta, \tau)\right],
\end{aligned}
$$

where

$$
\begin{aligned}
u_{1}(\eta, \tau) & =\left(\eta+\frac{1}{2} \frac{P r \eta^{2}}{1+R a}\right) \operatorname{erfc}\left\{\frac{\eta \sqrt{P r}}{2 \sqrt{\tau(1+R a)}}\right\} \\
& -\sqrt{\frac{P r \tau}{\pi(1+R a)}} \eta e^{-\frac{P r \eta^{2}}{4 \tau(1+R a)}}, \\
u_{2}(\eta, \tau) & =\left(\tau+\frac{1}{2} \eta^{2}\right) \operatorname{erfc}\left\{\frac{\eta}{2 \sqrt{\tau}}\right\}-\eta \sqrt{\frac{\tau}{\pi}} e^{-\frac{\eta^{2}}{4 \tau}} .
\end{aligned}
$$




\section{NuSSElt Number AND SKIn Friction AT The Plate $\eta=0$}

In this section we have obtained the expressions for Nusselt number and skin friction at the plate $\eta=0$ with reference to ramped temperature. Denoting Nusselt number by $N u$ and skin friction by $\tau_{0}$, we have for ramped temperature

$$
\begin{aligned}
& N u=-\left.\frac{\partial \theta}{\partial \eta}\right|_{\eta=0} \\
& =2 \sqrt{\frac{P r}{\pi(1+R a)}}[\sqrt{\tau}-\sqrt{\tau-1} H(\tau-1)], \\
& \tau_{0}=-\left.\frac{\partial u}{\partial \eta}\right|_{\eta=0} \\
& =\frac{4 \sqrt{1+R a}}{3 \sqrt{\pi}(\sqrt{1+R a}+\sqrt{P r})}\left[\tau^{\frac{3}{2}}-(\tau-1)^{\frac{3}{2}} H(\tau-1)\right],
\end{aligned}
$$

and for constant wall temperature

$$
\begin{aligned}
& N u=\sqrt{\frac{P r}{\pi \tau(1+R a)},} \\
& \tau_{0}=\frac{2 \sqrt{(1+R a) \tau}}{\sqrt{\pi}(\sqrt{1+R a}+\sqrt{P r})} .
\end{aligned}
$$

It is evident from Eqs. (23) and (25) that for a given $\tau$ the Nusselt number $N u$ increases with increase in $\mathrm{Pr}$ for both ramped wall temperature as well as constant wall temperature while the results are reversed with increase in radiation parameter $R a$. Solutions (24) and (26) reveal that for a given $\tau$, the skin friction at wall decreases with increase in $P r$ whereas it increases with increase in radiation parameter $R a$ for both ramped temperature as well as constant wall temperature.

\section{RESUltS AND DisCUSSION}

To gain a perspective of the physics of the flow regime, we have numerically evaluated the effects of Prandtl number $P r$, radiation parameter $R a$ and time $\tau$, on the velocity $u(\eta)$ and the temperature $\theta(\eta)$. In Figs. $2-7$, the spatial distribution of velocity $u(\eta)$ and temperature $\theta(\eta)$ are plotted for various values of $\operatorname{Pr}, R a$ and $\tau$. In these figures, we have examined the flow pattern corresponding to the Prandtl number equal to 0.71 for air. Figure 2 shows that the velocity decreases with increase in Prandtl number $\mathrm{Pr}$. This indicates that in Fig. 2, an increase in Prandtl number leads to fall the thermal boundary layer flow for ramped temperature as well as isothermal case. In the case of a high temperature flow, the triatomic gas corresponds to the value of Prandtl number greater than unity with a decisive importance to an effect of thermal boundary layer flow. Figure 3 reveals that an increase in radiation parameter $R a$ leads to rise the thermal boundary layer flow due to frictional force near the bounding wall. This will generally happen in the case of a thermal boundary layer flow with the effect of Prandtl number to determine the transient approach on the flow behavior. It is seen from Fig. 4 that the velocities increase with increase in time $\tau$ for ramped temperature as well as isothermal cases. An interesting feature of velocity profiles on Fig. 4 indicates nearly flat for small values of $\tau$, but assumes parabolic shape near the plate as $\tau$ increases.

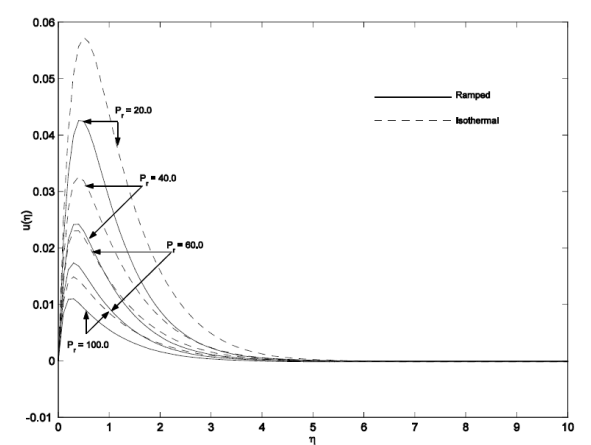

Fig. 2. Velocity profiles for four values of $\operatorname{Pr}$ with $R a=2$ and $\tau=2.0$.

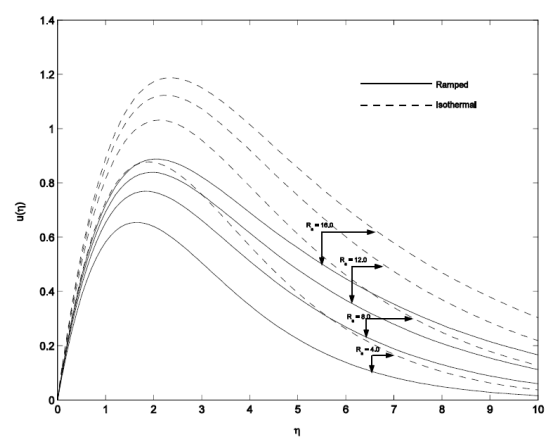

Fig. 3. Velocity profiles for four values of $R a$ with $\operatorname{Pr}=0.71$ and $\tau=2.0$.

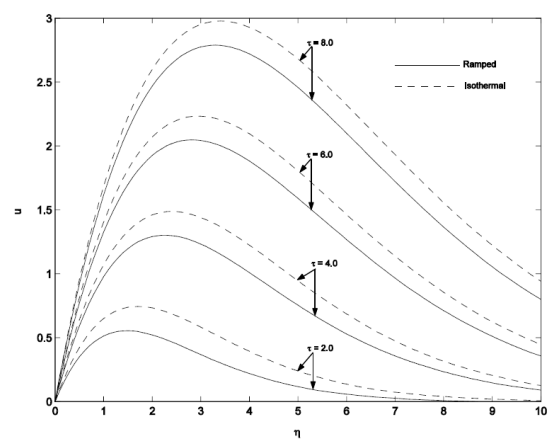

Fig. 4. Velocity profiles for four values of $\tau$ with $\operatorname{Pr}=0.71$ and $R a=2$.

Figure 5 demonstrates that in an optically dense medium its temperature decreases with increase in Prandtl number. It is interesting to note that for the case of higher Prandtl number $(\operatorname{Pr}>1)$ as for example of water $(P r=7)$, the diatomic molecules can be transformed into triatomic gases and the lighter particles leads to decrease the temperature due to absorption of scattering medium. Figure 6 reveals that the temperature increases with increase in radiation parameter. Viscous force accelerates thermal temperature of the fluid caused by friction to exert its influence of temperature profile as given by Fig. 6. It is noticed from Fig. 7 that the temperature increases with time $\tau$ due to moving layer of fluids. 


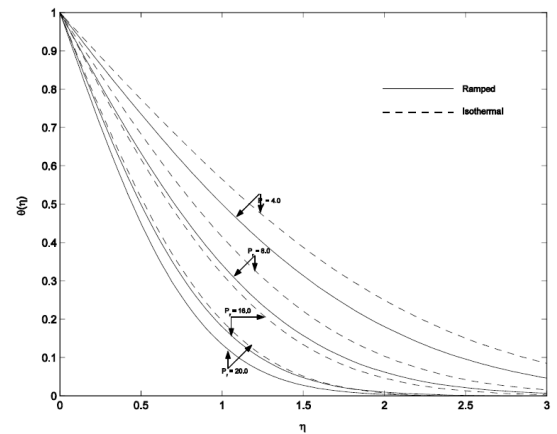

Fig. 5. Temperature profiles for four values of $\operatorname{Pr}$ with

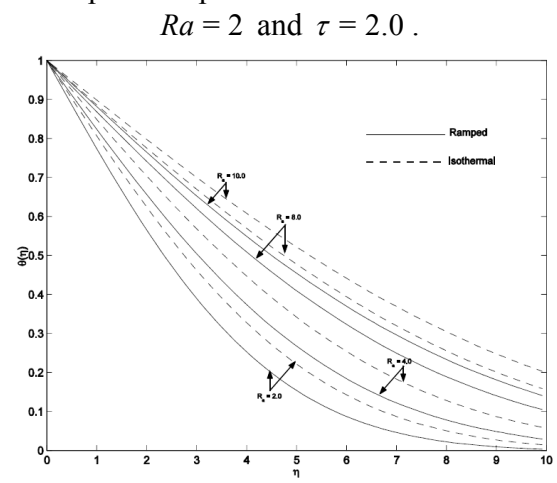

Fig. 6. Temperature profiles for four values of $R a$ with $\operatorname{Pr}=0.71$ and $\tau=2.0$.

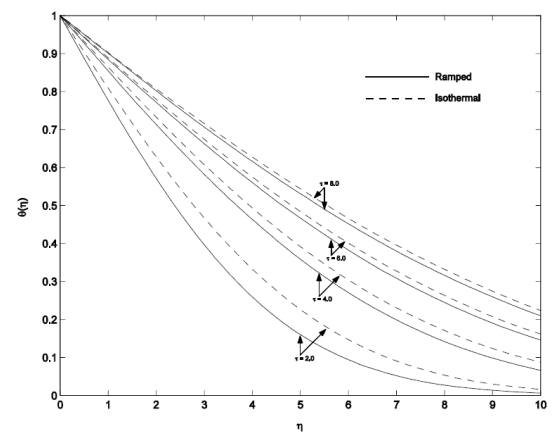

Fig. 7. Temperature profiles for four values of $\tau$ with $\operatorname{Pr}=0.71$ and $R a=2$.

\section{ConClusion}

A transient approach on radiation-convection heat transfer near a vertical plate with ramped wall temperature has been studied. This problem has been analyzed with a non-catalytic system of different Prandtl number of an optically dense medium with reference to diffusion concept of a thermal boundary layer flow. The soluion has been obtained by applying Laplace transform technique. It has been studied that two different solutions for fluid velocities out of which one valid for fluids of Prandtl number (Pr) different from $1+R a, R a$ being the radiation parameter and the other for which the Prandtl number equal to $1+R a$. The variation of velocities and fluid temperature are presented graphically.

\section{REFERENCES}

Bestman, A.R. and S.K. Adiepong (1988). Unsteady hydromagnetic free convection flow with radiative heat transfer in a rotating fluid. Astrophysics and Space science 143, 73-80.

Brewster, M.Q. (1992). Thermal Radiative Transfer and Properties. John Wiley and Sons, Inc., New York.

Cess, R.D. (1966). The interaction of thermal radiation with free convection heat transfer. Int. J. Heat Mass Transfer 9, 1269-1277.

Cheng, P. (1965). Study Of the Flow Of a Radiating Gas by a Differential Approximation. Ph. D. thesis, Standford University, California, U.S.A.

Chandran, P., N.C. Sacheti and A.K. Singh (2005). Natural Convection near a vertical plate with ramped wall temperature. Heat Mass Transfer 41, 459-464.

England, W.G. and A.F. Emery (1969). Thermal radiation effects on the laminar free convection boundary layer of an absorbing gas. Journal of Heat Transfer 91, 37- 44.

Ghosh, S.K. and I. Pop (2007). Thermal radiation of an optically thick gray gas in the presence of indirect natural convection. International Journal of Fluid Mechanics Research 34(6), 515-520.

Hottel , H.C. and A. Sarofim (1967). Radiation Heat Transfer, MacGraw Hill.

Hossain, M.A. and H.S. Takhar (1996). Radiation effect on mixed convection along a vertical plate with uniform surface temperature. Heat and Mass Transfer 31, 243-248.

Isachenko, V.P., V.A. Osipova and A.S. Sukomel (1969). Heat Transfer, Mir Publishers, Moscow.

Muthucumaraswamy, R. and P. Ganesan (2003). Radiation effects on flow past an impulsively started infinite vertical plate with variable temperature. Int. J. Applied Mechanics and Engineering 8(1), 125-129.

Naroua, H., P.C. Ram, A.S. Sambo and H.S.Takhar (1998). Finite element analysis of natural convection flow in a rotating fluid with radiative heat transfer. Journal of Magnetohydrodynamics and Plasma Research 7(4), 257-274.

Raptis, A. and C. Perdikis (2003). Thermal radiation of an optically thin gray gas. Int. J. of Applied Mechanics and Engineering 8(1), 31-134.

Takhar, H.S. and R.S.S. Gorla and V.M. Soundalgekar (1996). Radiation effects on MED free convection flow of a radiating gas past a semi-infinite vertical plate. Int. J. Numerical Methods Heat Fluid flow $6,77-83$. 